\title{
Engineering physical and chemical properties of softwood kraft lignin by fatty acid substitution
}

\author{
Siddhesh N. Pawar, Richard A. Venditti*, Hasan Jameel, \\ Hou-Min Chang and Ali Ayoub
}

Department of Forest Biomaterials, College of Natural Resources, North Carolina State University, Raleigh, North Carolina 27695-8005

\section{Corresponding Author}

Richard A Venditti

Department of Forest Biomaterials

College of Natural Resources

North Carolina State University

Raleigh, North Carolina 27695-8005

Tel: +1 9195156185

Fax: +19195156302

Email: richard_venditti@ncsu.edu 


\begin{abstract}
A process to attach fatty acids to lignin is reported which alters its thermal behavior. By attaching saturated $\mathrm{C}_{18}$ fatty acids to $\mathrm{OH}$ groups, stable lignin stearates (LS) of controllable degrees of substitution (DS) were synthesized. Interesting physical properties were observed, wherein LS was observed to melt and flow at temperatures as low as $50^{\circ} \mathrm{C}$. Melting was possible due to the crystallization of stearate chains when LS was purified by precipitation. A NMR method was established for quantification of the degree of substitution. At very high \%DS values (close to $100 \%$ ), the melting phenomenon was reversible, but at low \%DS, melting occurred only during the $1^{\text {st }}$ heating ramp during thermal cycling. The ability of LS to plasticize polystyrene (PS) is reported wherein integral blend films containing up to $25 \%$ by weight of LS were formed. The $\mathrm{T}_{\mathrm{g}}$ of the blended films could be lowered by $22^{\circ} \mathrm{C}$ using LS relative to PS. Lignin stearates have the potential to serve as interesting compounds for their ability to plasticize not only PS but other thermoplastic materials as well.
\end{abstract}




\section{Graphical abstract}

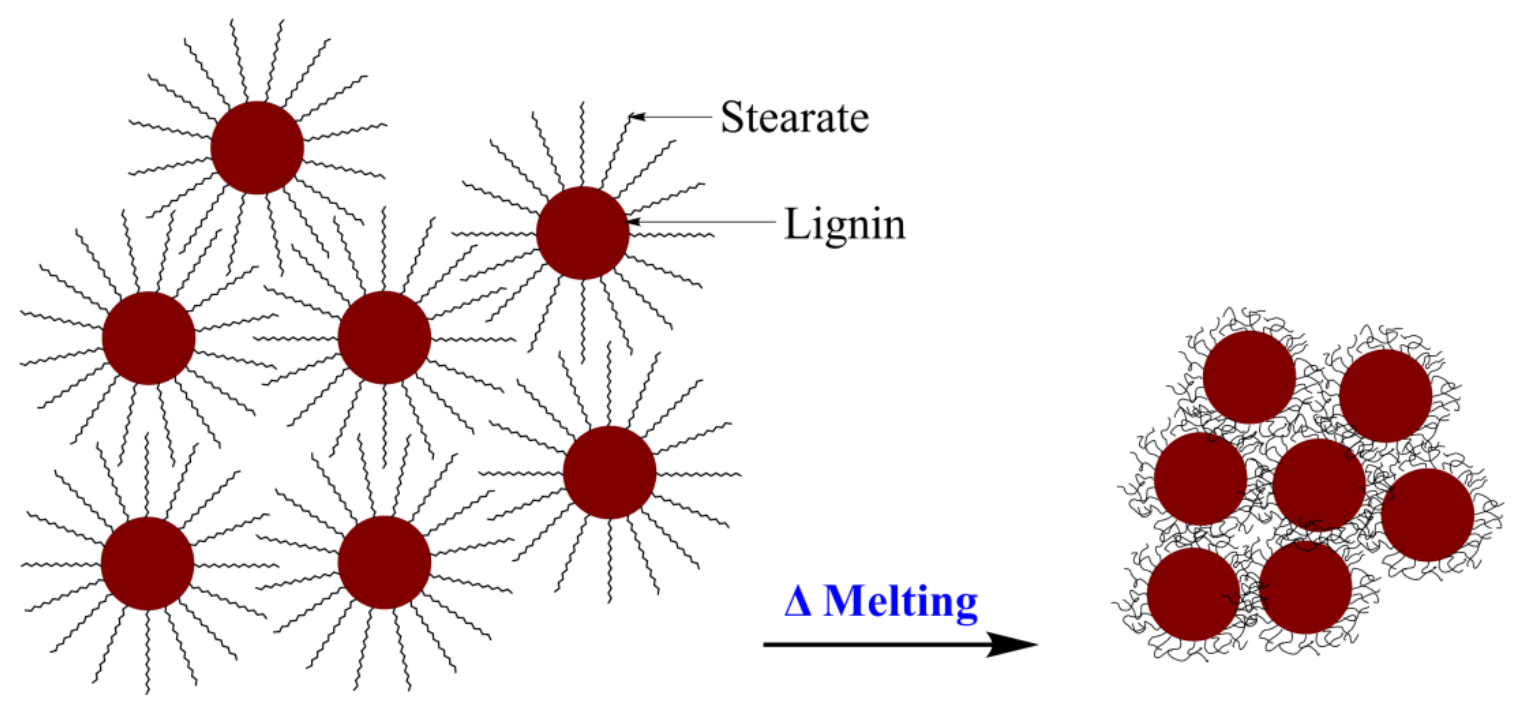

This research focuses on the chemical modification of lignin with stearate of controlled degree of substitution 


\section{Keywords}

Lignin, softwood, wood products, fatty acid, glass transition, melting

\section{Introduction}

Lignin is an important component of biomass, both in terms of its mass contribution and functionality. Lignin's structure as part of the wood composite is a topic of intense scientific debate. For example, while it is widely reported in the literature as a cross-linked network polymer, a recent report indicated to lignin being a linear oligomer (Crestini et al., 2011 and Mariotti et al., 2014 and Hulin et al., 2015). The pulp and paper industry is estimated to produce more than 50 million tons of lignin annually, most of which is burnt off to meet the energy demands of the pulp mills (Gosselink et al., 2004). Lignin when used as a fuel yields a value equivalent of $\$ 0.18 / \mathrm{kg}$. However, if converted to high-value products, the value equivalent can potentially be raised up to $\$ 1.08 / \mathrm{kg}$ (Vishtal and Kraslawski, 2011). Therefore, there is enormous interest in transforming lignin to attain properties competitive with commercial high volume polymers such as polyethylene (PE), polypropylene (PP), polystyrene (PS) and polyvinyl chloride (PVC). Factors influencing the physicochemical properties of lignin are the type and specie of woody or non-woody biomass, the technical process used for pulping, and the method used to separate lignin from black liquor. Depending on these factors, technical lignins may contain varying amounts of methoxyl, phenolic hydroxyl, primary and secondary aliphatic hydroxyl, carbonyl and carboxyl groups. In this study we shall focus on utilization of the hydroxyl groups for lignin modification. 
Several ways of modifying lignin via hydroxyl group reactions were previously reported. Cui et al., 2013 described methylation of lignin using dimethyl sulfate or methyl iodide to create a lignin based thermoplastic material. Laurichesse et al., 2014 summarized most of the applications based on esterified lignins. The applications were devoted to the development of elastomeric products, epoxy resins and polyesters. Sailaja et al., 2010 developed a new biobased composites of polyethylene and esterified lignin. The esterification of lignin has been used to enhance interfacial adhesion where the tensile strength of the blends improved with values approaching close to that of neat polyethylene.

Glasser et al., 1984 previously reported the hydroxyalkylation of lignin by reaction with alkylene oxides to create engineering plastics. Hydroxypropyl lignin (HPL) derivatives were subsequently epoxidized and crosslinked networks formed using aromatic diamines as curing agents (Kelley et al., 1988). Saraf and Glasser, 1984 also described lignin based polyurethane films using HPL reaction with diisocyanates. To improve stretching, polyethylene glycol (PEG) and poly(butadiene glycol) extended polyurethanes were also reported (Saraf et al., 1985). In addition to polymeric modification of the hydroxyl groups, simple acetylation procedures involving acetic anhydride and pyridine are routinely performed in laboratories for lignin analysis (Capanema et al., 2004). These types of chemical modification derivatives improve the solubility of the lignin in organic solvents and some other physical properties as Laurichesse et al., 2014 reported.

Recently, a solvent less system comprising of softwood kraft lignin and styrene monomer was subjected to $\gamma$-irradiation to prepare polystyrene grafted lignin derivatives via radical chemistry (Ayoub et al., 2014). This work has demonstrated that the effect of irradiation to make the 
material more thermally stable relative to lignin alone, thus improving its processability at hjigh temperatures.

While the lignin modification literature is vast, large scale commercialization of lignin based products has been stifled due to supply chain issues and poor mechanical and thermal properties relative to synthetic polymers (Vishtal and Kraslawski, 2011).

A survey of the patent literature showed a recent invention in which acetylated lignin was reacted with tall oil fatty acids to obtain fatty acid esters of lignin, as acetic acid was distilled off during reaction (Pietarinen et al., 2014). These new derivatives were reported to be more hydrophobic and possessed low melting points. A proceedings article briefly described the reaction of lignin with fatty acid chlorides but did not present resulting thermal properties (Mariotti et al., 2012). Later on, Mariotti et al., 2014 reported a new wood plastic composite while exploring for the first time the potential of using esterified kraft lignin as a coupling agent. Two types of lignin esterification with maleic and succinic acids anhydrides were explored. An improved of the mechanical property was presented. Hulin et al., 2015 described for the first time a new method of lignin esterification with long acyl chains in ionic liquids. The chemical esterification improved the miscibility of lignin in apolar polyolefin matrices. The final product exhibited interesting thermal and textural properties comparing to the kraft lignin.

Historically, the idea of reacting fatty acids with lignin has been explored by Lewis et al., 1943. Additionally, the esterification of lignin with tall oil fatty acids (containing 3.5\% saturated and 86.3\% unsaturated fatty acids) for water vapor and oxygen barrier coatings has been previously described (Hult et al., 2013). That study reported that the glass transition temperature could not 
be observed for esterified samples. The $\%$ mass addition of fatty acids was around $0.1 \%$ for that report. Our study herein, advances this research in that it shows the synthesis of lignin esters with mass addition of up to $100 \%$. Within this larger range of DS, this study shows a much more robust change in the thermal behavior with esterification of lignin, with the lignin esters showing interesting melting phenomena. In a related study, Sagar et al. 1995 examined the properties of fatty-acid esters of starch and found that the fatty acids acted as internal plasticizers, with larger ester groups making the material more melt processable and ductile.

In this study, we explore the reaction, characterization and applicability of lignin esters to use as compatibilizers or plasticizers, not described previously. Further, we show the changes in thermal properties with degree of substitution of fatty acids, not previously reported. It has become of great interest to develop compatible blends of synthetic and natural polymers such that properties like biodegradability or water uptake can be modified. We describe the synthesis of fatty acid esters of non-acetylated commercial softwood kraft lignin using acid chlorides. Depending on the type of fatty acid chain attached, interesting thermal and physical properties of the lignin esters can be expected. While a commercial fatty acid chloride, stearoyl chloride (StCl) was used in the study, other saturated or unsaturated acid chlorides can also be used to react with lignin. Products with varying degrees of substitution (DS) were prepared. A ${ }^{1} \mathrm{H}-\mathrm{NMR}$ method for quantifying the addition of fatty acid chains attached to the lignin molecule is described. Thermal analysis was performed using TGA and DSC. Finally, compatibility of the derivatives with polystyrene (PS) and their ability to plasticize PS is reported for the first time.

\section{Materials and methods}

\subsection{Materials.}


Biochoice $^{\mathrm{TM}}$ (BCL) softwood Kraft Lignin was provided by Domtar. Chemical composition of BCL was as follows: lignin $=98.2 \%$, arabinan $=0.2 \%$, galactan $=0.7 \%$, glucan $=0.1 \%$, xylan $=0.4 \%$, and ash $=0.73 \%$, with $\mathrm{pH}=3.9$. Molecular weight $=5500 \mathrm{~g} / \mathrm{mol}$. Elemental composition was as follows: Methoxyl content $=13.8 \%$; Carbon $=64.4 \%$; Hydrogen $=6.24 \%$; Oxygen $=27.9 \% ; \quad$ Nitrogen $=0.36 \% ; \quad$ Sulfur $=1.36 \% . \quad$ Molecular $\quad$ formula of $\quad \mathrm{C}_{9} \quad$ unit $=\mathrm{C}_{9} \mathrm{H}_{8.93} \mathrm{O}_{2.37}\left(\mathrm{OCH}_{3}\right)_{0.814} \mathrm{~S}_{0.079}$ with an average $\mathrm{M}_{\mathrm{W}}$ of $182.6 \mathrm{~g} / \mathrm{mol}$; Quantitative ${ }^{13} \mathrm{C}-\mathrm{NMR}$ analysis yielded the following groups per 100 aromatic rings: 5-5' ether $=31.8, \beta-1=1.2, \beta-5=$ 4.0, primary aliphatic $\mathrm{OH}=26.6$, secondary aliphatic $\mathrm{OH}=17.6$, phenolic $\mathrm{OH}=49.4$, total etherified $=52.0$, methoxyl $=63.2, \mathrm{C}_{\gamma}$ in $\beta-\mathrm{O}-4$ without $\mathrm{C}_{\alpha}=\mathrm{O}=11.5$, aliphatic $\mathrm{COOR}=8.7$, conjugated $\mathrm{COOR}=4.3$, and degree of condensation $=71.4$. Stearoyl chloride $(\mathrm{St}-\mathrm{Cl})$, pyridine (Pyr), 1,4-dioxane, methanol, reagent alcohol, hexane, acetone, chloroform, $\mathrm{KBr}, \mathrm{CDCl}_{3}$, DMSO- $\mathrm{d}_{6}$ and polystyrene (PS) were obtained from Sigma-Aldrich (St. Louis, MO, USA). All chemicals were used as purchased except 1,4-dioxane, which was distilled over $\mathrm{NaOH}$ and stored under $\mathrm{N}_{2}$.

\subsection{Synthesis of lignin stearate (LS)}

Lignin $(2 \mathrm{~g})$ was weighed into a 3-neck flask. A volume of $50 \mathrm{~mL}$ dioxane was added and stirred at room temperature for roughly $2-3$ hours under $\mathrm{N}_{2}$ to dissolve the lignin. The required amounts of St-Cl and Pyr (calculated based on total $\mathrm{OH}$ groups available) were then added to the flask and stirred at $80^{\circ} \mathrm{C}$ for roughly 18 hours. Following the reaction, the mixture was added dropwise to a suitable precipitating solvent, either hexane or ethanol, see Table 1. The crude solid was then filtered under vacuum and recovered. To further purify the crude product, it was washed in a Soxhlet extractor overnight using a suitable extraction solvent. Generally, the 
precipitation solvent was the same as extraction solvent. The choice of precipitation and extractions solvent depended on the amount of $\mathrm{St}-\mathrm{Cl}$ added for reaction (Table 1). After extraction, the solid was dried in air first and then under vacuum at room temperature.

\section{$2.3{ }^{1} H-N M R$.}

Analysis was performed using a Bruker $300 \mathrm{MHz}$ NMR with manual lock and shim. Choice of the NMR solvent was dictated by the amount of stearate substitution. Products with higher DS dissolved in $\mathrm{CDCl}_{3}$, while those with lower DS were soluble in DMSO-d $\mathrm{d}_{6}$. For acquisition, 6-10 $\mathrm{mg}$ solid was weighed and dissolved in $0.6 \mathrm{~mL}$ of solvent and added to a $5 \mathrm{~mm}$ NMR tube. Acquisition was performed at room temperature and 164 scans were obtained. Data analysis was performed using MestReNova LITE v. 5.2.5.

\subsection{FTIR analysis}

Analysis was performed using a Perkin Elmer Frontier instrument in transmission mode. An amount of $200 \mathrm{mg}$ of $\mathrm{KBr}$ was mixed with $30-40 \mathrm{mg}$ of lignin stearate and ground together using a mortar-pestle. The mixture was then pelletized using a Perkin Elmer 15 ton manual hydraulic press. The number of scans obtained for each measurement was 32 .

\subsection{Thermogravimetric analysis (TGA)}

Thermogravimetric measurements were performed using a TA Q500 instrument (TA, New Castle, DE) instrument loaded with a platinum pan. Sample amounts ranged between 5-10 mg under $\mathrm{N}_{2}$ atmosphere. Heating rate employed was $10{ }^{\circ} \mathrm{C} / \mathrm{min}$ from $40-600{ }^{\circ} \mathrm{C}$. Data analysis was performed using Universal Analysis 2000, 4.5.0.5. 


\subsection{Differential scanning calorimetry (DSC)}

Measurements were performed using TA Q100 instrument (TA, New Castle, DE) equipped with a chiller. Sample amounts ranged between 5-10 mg and the analysis was carried out under $\mathrm{N}_{2}$ atmosphere using aluminum hermetic pans with a hole punched to facilitate moisture removal. The experimental protocol used was as follows: (A) Heat to $105{ }^{\circ} \mathrm{C}$ at $10{ }^{\circ} \mathrm{C} / \mathrm{min}$, and hold isothermally for $20 \mathrm{~min}$ to completely remove moisture. (B) Cool to $40{ }^{\circ} \mathrm{C}$ at $10 \mathrm{C} / \mathrm{min}$. This completes heating cycle number 1 . (C) Heat to $180{ }^{\circ} \mathrm{C}$ at $10{ }^{\circ} \mathrm{C} / \mathrm{min}$. (D) Cool to $40{ }^{\circ} \mathrm{C}$ at 10 ${ }^{\circ} \mathrm{C} / \mathrm{min}$. This completes heating cycle number 2. (E) Heat to $180{ }^{\circ} \mathrm{C}$ at $10^{\circ} \mathrm{C} / \mathrm{min}$. (F) Cool to 40 ${ }^{\circ} \mathrm{C}$ at $10{ }^{\circ} \mathrm{C} / \mathrm{min}$. This completes heating cycle number 3 . A slightly modified protocol was used for PS blend films as follows: (A) Heat to $180{ }^{\circ} \mathrm{C}$ at $10{ }^{\circ} \mathrm{C} / \mathrm{min}$, and hold isothermally for $5 \mathrm{~min}$. (B) Cool to $20^{\circ} \mathrm{C}$ at $10{ }^{\circ} \mathrm{C} / \mathrm{min}$. (C) Heat to $180{ }^{\circ} \mathrm{C}$ at $10{ }^{\circ} \mathrm{C} / \mathrm{min}$. $\mathrm{T}_{\mathrm{g}}$ 's were measured during the second heating scan. Data analysis was performed using Universal Analysis 2000, build 4.5.0.5

\subsection{LS-PS blends}

Mixtures of LS and PS were prepared with LS contents of 0, 5, 25, 50, 75 and $100 \%$. A total of $200 \mathrm{mg}$ of solid (LS + PS) was weighed for each mixture. The mixtures were then dissolved for approximately 24 hours in $1 \mathrm{ml}$ solvent which contained a 50-50 mixture of acetone $+\mathrm{CHCl}_{3}$. As a control, $200 \mathrm{mg}$ of solid (BCL + PS) was weighed for each mass \% mixture and dissolved in 1 $\mathrm{mL}$ 1,4-dioxane. Thereafter, the solutions were placed in silicone molds (roughly $1 \frac{1}{2} 2$ inch diameter), covered with aluminum foil and dried at room temperature until most of the solvent had evaporated. The molds were then placed in a vacuum chamber at room temperature for complete drying. 


\section{$2.8 X$-ray diffraction $(X R D)$}

Measurements were performed using a PANalytical Empyrean X-Ray diffractometer with linear detector and non-ambient environment at $40 \mathrm{kV}$ voltage and $25 \mathrm{~mA}$. Scanned angle was set between $5-33^{\circ}$.

\subsection{Scanning electron microscopy (SEM)}

Morphologies were examined using a FEI XHR-Verios 460L scanning electron microscope. Powdered samples were deposited on a carbon tape placed on a stage, with the excess being blown off using a jet of dry $\mathrm{N}_{2}$ gas. A concentric backscatter detector was used to obtain high quality images.

\section{Results and discussion}

\subsection{Lignin esters synthesized from saturated fatty acid chloride $\left(C_{18}\right)$}

Of the functional groups generally present in lignin, hydroxyl groups are abundant and easily accessible to reagents. While $\mathrm{OH}$ groups are not among the most reactive species, a good way to get reaction products with high conversions is by using reactive reagents. Esterification reactions are a common way to react $\mathrm{OH}$ groups. Typically encountered reagents to achieve these reactions can be carboxylic acids, and their acid chlorides and anhydrides. Of the three, carboxylic acids are least reactive. Acid chlorides and anhydrides can react rapidly with free hydroxyls to yield esters. Since the objective of this study was to synthesize fatty acid esters, we considered the use of both fatty acid chlorides and anhydrides as reagents. Commercial acid 
chlorides were significantly less expensive relative to anhydrides and were therefore selected. Additionally, this project was limited to the synthesis and property evaluation of lignin esters synthesized from stearoyl chloride $-\mathrm{a}_{18}$ saturated fatty acid chloride.

The reaction procedure employed in the synthesis of lignin stearates first involved the dissolution of lignin in a suitable non-aqueous medium. Homogeneous dissolution is known to allow better accessibility to the reactive functional groups relative to heterogeneous mixtures (Pawar et al. 2011). For this study, we were able to dissolve softwood kraft lignin in 1,4-dioxane at a concentration of $40 \%(\mathrm{w} / \mathrm{v})$. Upon dissolution, the desired amount of the reagent $\mathrm{St}-\mathrm{Cl}$ was added. Figure 1 shows the reaction. Molar calculations required an estimation of the number of $\mathrm{OH}$ groups available in lignin. ${ }^{13} \mathrm{C}-\mathrm{NMR}$ studies revealed that $93.6 \pm 3 \mathrm{OH}$ groups were present per 100 aromatic rings. Additionally, the average molar mass of the $\mathrm{C}_{9}$ residue for lignin was 182 $\mathrm{g} / \mathrm{mol}$. Based on this information, the molar equivalents of $\mathrm{St}-\mathrm{Cl}$ relative to available $\mathrm{OH}$ groups were calculated and added to the reaction mixture. It was presumed that both phenolic as well as aliphatic groups reacted with the acid chloride. A reaction temperature of $80^{\circ} \mathrm{C}$ was used to prevent thermal condensation reactions due to radical formation at higher temperatures. Prior to scale up of this process, considerations about reaction times and solvents and washing processes are needed.

\subsection{Solubility}

Reaction workup entailed choosing an appropriate solvent for precipitation and extraction. As expected, upon derivatization with stearate esters, the polarity index of lignin decreases. The index of polarity decreases are reflected in the solubility characteristics shown in Table 1 . At higher DS values, lignin stearate becomes fully soluble in solvents such as hexane or chloroform, 
but insoluble in polar solvents such as dimethylsulfoxide (DMSO). While at lower DS values, complete solubility in polar solvents such as DMSO was observed; LS was insoluble in hexane or chloroform. This change in the hydrophilicity-hydrophobicity balance of the lignin esters dictates the solvents used during workup with hexane being used for low DS and ethanol being used for high DS materials.

\subsection{Chemical characterization}

Chemical characterization of products was performed using ${ }^{1} \mathrm{H}-\mathrm{NMR}$ and FTIR. Lignin prior to fatty acid derivatization is soluble in DMSO- $\mathrm{d}_{6}$. Similarly, lignin stearates with low DS values were dissolved in DMSO- $\mathrm{d}_{6}$ for NMR analysis. Products with higher degrees of substitution dissolved in $\mathrm{CDCl}_{3}$. Figure 2 shows a comparison of the ${ }^{1} \mathrm{H}-\mathrm{NMR}$ spectra of $\mathrm{BCL}$ and the corresponding lignin stearate formed upon derivatization. In the lignin spectrum, two broad peaks can be observed - aromatic protons appear around $7.0 \mathrm{ppm}$ while methoxyl protons are observed around $3.5 \mathrm{ppm}$. Upon stearate derivatization, additional signals arising from the stearate protons appear in the region between 0.5-3.0 ppm.

The FTIR spectra in Figure 3 show a comparison of lignin and fully derivatized lignin stearate (LS-97\% from Table 1). Non-derivatized lignin shows a broad OH stretching vibration around $3390 \mathrm{~cm}^{-1}$. Upon derivatization, the $\mathrm{OH}$ stretching disappears, and two new sets of peaks appear - aliphatic $\mathrm{C}-\mathrm{H}$ stretching from the stearate groups $\left(2918,2850 \mathrm{~cm}^{-1}\right)$ and ester $\mathrm{C}=\mathrm{O}$ stretching vibration $\left(1740,1762 \mathrm{~cm}^{-1}\right)$. This evidence strongly supports the formation of stearate esters of lignin.

${ }^{1} \mathrm{H}-\mathrm{NMR}$ is a tool that can be used to measure the DS value of lignin esters. Because stearate proton signals are separated from lignin, they can be integrated relative to a standard. Known 
amounts of standards such as tetramethylsilane (TMS) or 2,3,4,5,6-pentafluorobenzaldehyde (PFB) may be added externally to the NMR tube. The stearate peaks can be integrated relative to the peaks arising from the standards. DS can then be measured in terms of the number of stearate groups per gram of LS sample. However, several issues may arise in using an external standard such as accurate measurement of standard to sample mass, volatility and the use of an appropriate $\mathrm{d} 1$ relaxation delay parameter.

To circumvent these problems, the methoxyl peaks of lignin were used as an internal standard to calculate DS by peak integration. Methyl groups are linked to the lignin aromatic rings via ether groups. Under the conditions used for reaction and workup, the ether groups are expected to remain intact. The number of methoxyls per 100 aromatic rings was 63.2 , as calculated using ${ }^{13} \mathrm{C}$-NMR. A method previously described in the literature was used for this calculation. ${ }^{12}$ This number was thus expected to stay constant even as lignin was converted to lignin stearate. Therefore, by integrating the methoxyl region in the ${ }^{1} \mathrm{H}-\mathrm{NMR}$ spectrum (3.5-4.5 ppm) relative to the stearate signals $(0.5-3.0 \mathrm{ppm})$, DS was calculated as the number of stearate groups per 100 aromatic rings. Relaxation delay studies were performed and a d1 value of 1.0 was found to be suitable for quantitative measurements. Table 1 describes how the DS was controlled by varying the molar equivalents of $\mathrm{St}-\mathrm{Cl}$ and pyridine in the reaction. The mass percent addition to the lignin could be extremely high, with a maximum of $97 \%$ for these reaction conditions, significantly higher than produced in a previous study (Hult et al., 2013). One caveat of this DS measurement method however, is that the number of methoxy groups will vary for differing lignins depending on plant resource and isolation methodology used and this must be accounted for in comparisons of different lignins.

\subsection{Thermal analysis}


Thermal analysis was performed using TGA (Table 2) and DSC (Table 3). Moisture contents of LS were measured by TGA by observing the mass loss around $100{ }^{\circ} \mathrm{C}$ and compared to those of BCL. As expected, BCL being more polar in character contained the highest amount of moisture. For substituted materials, the moisture content was lower with increased DS values, as expected. In addition to the decrease of moisture occurring on heating up to around $100{ }^{\circ} \mathrm{C}$, residual mass was recorded upon completion of the TGA experiment. $\mathrm{BCL}$, when heated up to $600{ }^{\circ} \mathrm{C}$, yielded a residual mass of $42.67 \%$. This value was relatively high compared to LS. Since BCL is composed of mostly aromatic structures, it has relatively higher thermal stability. Upon stearate substitution above $10 \%$, significant mass contribution from the long aliphatic chains was observed. The stearate chain has a molar mass of $267 \mathrm{~g} / \mathrm{mol}$, while the lignin $\mathrm{C}_{9}$ unit is 182 $\mathrm{g} / \mathrm{mol}$. Because the long aliphatic chains can thermally degrade relatively more facile and are expected to produce char less compared to the aromatic backbone of lignin, the residual masses obtained for LS are generally lower. To support this, we performed TGA analysis on stearic acid which yielded $0 \%$ residual mass. As shown in Table 2, for the almost fully substituted LS-97\%, the residual mass can be as low as $17 \%$. Based on the knowledge of the stearate mass contributions in LS, expected residual masses were calculated for comparison with the TGA results, Table 2 . We assumed that $42.67 \%$ mass of the lignin fraction, and $0 \%$ mass of the stearate fraction remained after heating up to $600{ }^{\circ} \mathrm{C}$. The calculated residual mass values show good correlation with those from TGA, especially at high DS values. At low DS, a small difference in the calculated and experimentally measured residual mass values was observed. Nevertheless, this points to the fact that the stearate chains thermally decompose and do not form char as much as lignin. It also is in agreement with reported DS values. All TGA plots are provided in Supporting Information Figure S1. The plots show that the higher DS materials have 
a steep mass loss versus temperature result around $400^{\circ} \mathrm{C}$ whereas the low DS materials have a more gradual mass loss over the range of $250-400^{\circ} \mathrm{C}$.

DSC analysis of BCL was performed according to a procedure that is typically used to measure the glass transition temperature, $\mathrm{T}_{\mathrm{g}}$, of kraft lignin, Table 3. Initially, lignin was heated to $105{ }^{\circ} \mathrm{C}$ in order to remove moisture. Thereafter, lignin was heated up to $180{ }^{\circ} \mathrm{C}$. For BCL, the $\mathrm{T}_{\mathrm{g}}$ appeared at $144{ }^{\circ} \mathrm{C}$, as shown in Figure 4 . When stearates were substituted into lignin however, interesting behavior was observed. For LS-97\% with heating and cooling rates of 10 ${ }^{\circ} \mathrm{C} / \mathrm{min}$, a melting endotherm was observed in the 1 st heating scan to $180{ }^{\circ} \mathrm{C}$ with $\mathrm{T}_{\mathrm{m}}=46{ }^{\circ} \mathrm{C}$. In

the $2^{\text {nd }}$ heating scan to $180{ }^{\circ} \mathrm{C}$, the melting point was lowered to $\mathrm{T}_{\mathrm{m}}=31{ }^{\circ} \mathrm{C}$. For $\mathrm{LS}-90 \%$ with heating and cooling rates of $10^{\circ} \mathrm{C} / \mathrm{min}$, a melting endotherm was observed in the $1^{\text {st }}$ heating scan with $\mathrm{T}_{\mathrm{m}}=48^{\circ} \mathrm{C}$. In the $2^{\text {nd }}$ heating scan however, no (or extremely weak) melting endotherm was observed. This type of behavior was intriguing, wherein at DS values nearing $100 \%$, the melting process was largely reversible with endotherms observed in the $2^{\text {nd }}$ heating scans. At lower DS values (90\% or lower), melting was irreversible wherein no endotherms were observed during the $2^{\text {nd }}$ heating scans. Visual observations of the viscosity of the materials above these melting points showed the materials to have water-like viscosities. Figure S2 and S3 in Supporting Information shows comparisons of endotherms observed in the $1^{\text {st }}$ and $2^{\text {nd }}$ heating scans respectively, for all LS samples reported in Table 1. The melting points observed for all LS samples are reported in Table S1 of Supporting Information.

\subsection{Crystallinity and morphology}

The melting phenomena observed in the $1^{\text {st }}$ heating scan but not in the $2^{\text {nd }}$ heating scan of LS was probed further. Possible stearate crystallization was suspected to be occurring as LS was 
precipitated during reaction workup. These crystals likely melted upon heating. In order to confirm this, XRD measurements were performed on LS-90\% prior to melting (LS Pre-melt) and after melting (LS Post-melt). The plots are shown in Figure 5. The peaks appearing at $2 \theta \approx 7^{\circ}$ in $\mathrm{XRD}$ are from the $\mathrm{X}$-ray window on the instrument. BCL is amorphous, and as such, shows no crystalline peaks. LS Pre-melt however shows a clear crystalline peak appearing at $2 \theta \approx 22^{\circ}$. The same LS was then melted on a hot plate by heating to $80{ }^{\circ} \mathrm{C}$ and allowed to cool to room temperature. The sample was then crushed and its XRD pattern was observed. The sharp crystalline peak does not appear in LS Post-melt in Figure 5. This observation was in agreement with the statement that the stearate chains crystallize upon precipitation, but melt irreversibly, perhaps do to a rearrangement at the higher temperatures in which the lignin segments interfere with the ordering. Figure 6 shows a schematic representation of the melting of crystalline stearate chains, as well as images of lignin stearate pre- and post-melt.

SEM imaging was performed to study the morphology of the BCL compared to LS-90\% premelt and post-melt, Figure 7. Both BCL and LS Pre-melt were powders with fine particle size. Since sample preparation involved deposition on carbon tapes, these samples were easier to handle. LS Post-melt on the contrary was difficult to crush into a fine powder since it was somewhat tacky at room temperature. It was therefore crushed into fairly large sized chunks for imaging. Furthermore, during imaging, LS Post-melt showed stronger insulating behavior relative to BCL and LS Pre-melt. This presented a great challenge in acquiring images at higher magnification levels. We therefore used 10,000 X magnification to compare the three samples. BCL particles were highly porous. When transformed into LS Pre-melt, the particles were relatively less porous. When melted and cooled back down to LS Post-melt, a very dense material was formed which showed no porosity. It is interesting to note that while LS Pre-melt 
and Post-melt are chemically alike, a single melt-cool cycle transforms its physical characteristics drastically from a porous, crystalline substance to a non-porous and amorphous one.

\subsection{Lignin stearate blends with polystyrene}

In order to study the applicability of LS in blending with PS, blends of PS with BCL, LS-46\% and LS-97\% were prepared by solvent casting. DSC experiments were designed such that the films were heated to $180{ }^{\circ} \mathrm{C}$ in the $1^{\text {st }}$ heating scan before cooling to $20^{\circ} \mathrm{C}$. The transitions occurring in the $2^{\text {nd }}$ heating scans were then studied. As mentioned previously, LS shows crystalline behavior when precipitated or dried from solvents. True blends with PS were formed only when the films were heated in the $1^{\text {st }}$ scan above the softening temperatures of the respective components. Measuring the transitions in the $2^{\text {nd }}$ scan therefore allowed accurate $T_{g}$ determinations. Additionally, the blended films were in intimate contact with the bottoms of the DSC pans during the 2nd scan which reduced noise in the thermograms. The $T_{g}$ and change in heat capacity at the glass transition, $\Delta \mathrm{C}_{\mathrm{p}}$, values are reported for 5 and $25 \%$ weight addition of LS in Table 3. All DSC thermograms are depicted in Figures S4-S19 of Supporting Information.

PS film cast from an acetone solution showed a $\mathrm{T}_{\mathrm{g}}=99{ }^{\circ} \mathrm{C}$ with an associated $\Delta \mathrm{C}_{\mathrm{p}}=0.242$ $\mathrm{J} / \mathrm{g} /{ }^{\circ} \mathrm{C}$. For the concentrations ranges studied, $5 \%$ and $25 \%$ blends yielded integral films for BCL, LS- $97 \%$ and LS-46\%. At weight ratios of $50 \%$ and above, the films were too brittle to show structural integrity. It is interesting to compare the thermal properties of all three lignin-PS mixtures at 25\% concentration. For BCL, LS- $46 \%$ and LS-97\%, the $\mathrm{T}_{\mathrm{g}}$ measured were 96,91 and $78{ }^{\circ} \mathrm{C}$ respectively The corresponding $\Delta \mathrm{C}_{\mathrm{p}}$ values for BCL, LS- $46 \%$ and $\mathrm{LS}-97 \%$ at $25 \%$ 
weight addition to PS, which provide a measure of the softening ability were 0.231 (5\% drop relative to pure PS), 0.193 (20\% drop relative to pure PS) and $0.218 \mathrm{~J} / \mathrm{g} /{ }^{\circ} \mathrm{C}(10 \%$ drop relative to pure PS). In the case of PS-LS-97\% blends, at LS concentration of 50\% and above, melting endotherms originating from LS-97\% persisted. This indicates that the lowering of the $T_{g}$ of PS at high concentrations of LS-97\% is not efficient, due to a lack of complete miscibility. A $\mathrm{T}_{\mathrm{g}}=$ $89^{\circ} \mathrm{C}$ is observed at $50 \%$ concentration, which is higher than the $\mathrm{T}_{\mathrm{g}}=78{ }^{\circ} \mathrm{C}$ for $\mathrm{LS}-97 \%$ at $25 \%$ concentration. PS blends with LS-46\% show similar behavior wherein there is a rise in $\mathrm{T}_{\mathrm{g}}$ above the $25 \%$ LS concentration.

Blends of PS with BCL $\left(\mathrm{T}_{\mathrm{g}}=144^{\circ} \mathrm{C}\right)$ showed unexpected behavior wherein lignin caused a depression in the $\mathrm{T}_{\mathrm{g}}$ of PS. This is unexpected and may be due to the lower molecular weight fractions of lignin (which can have $\mathrm{T}_{\mathrm{g}}$ 's well below that of PS) interacting with PS. However, at $25 \%$ additions to PS, BCL showed a much smaller $\mathrm{T}_{\mathrm{g}}$ depression than $\mathrm{LS}$, reflecting a significantly better plasticization ability of LS compared to BCL.

\section{Conclusion}

A strategy to attach fatty acid molecules to softwood kraft lignin using simple esterification chemistry was reported. Saturated $\mathrm{C}_{18}$ fatty acids were substituted on lignin to prepare lignin stearate, whereby the number of fatty acids attached can be controlled by varying the molar equivalents of reagent added. A ${ }^{1} \mathrm{H}-\mathrm{NMR}$ method was developed for quantification of the degree of substitution. Interesting physical properties were observed, wherein LS was observed to melt at temperatures as low as $50{ }^{\circ} \mathrm{C}$. At very high $\% \mathrm{DS}$ values (close to $100 \%$ ), the melting phenomenon was largely reversible, but at low $\% \mathrm{DS}$, melting occurred only during the $1^{\text {st }}$ heat. Melting originated from the crystallization of stearate chains when LS was purified by 
precipitation. When blends of PS with LS or with BCL at $25 \%$ concentration were compared, LS-97\% (LS with a $97 \%$ DS) was found to lower the $\mathrm{T}_{\mathrm{g}}$ of PS from $100{ }^{\circ} \mathrm{C}$ to $78{ }^{\circ} \mathrm{C}$, LS- $46 \%$ lowered the $\mathrm{T}_{\mathrm{g}}$ to $91{ }^{\circ} \mathrm{C}$, whereas BCL lowered the $\mathrm{T}_{\mathrm{g}}$ only slightly to $96{ }^{\circ} \mathrm{C}$, indicating better plasticization efficiency for the higher DS materials. At LS concentrations of up to $25 \%$ integral blend films can be formed in which the $\mathrm{T}_{\mathrm{g}}$ of PS can be lowered by up to $22^{\circ} \mathrm{C}$. Lignin stearates may therefore serve as interesting candidates for further studies on their ability to plasticize not only PS but other thermoplastics as well.

\section{Acknowledgments}

The authors wish to thank the United States Department of Agriculture (Grant number 1503/2011-0952) and Domtar Corporation for their sponsorship of this project. We would also like to thank Chuck Mooney for assistance with SEM, Chris Fancher for help with XRD and Justin Coor for assistance with the lab experiments.

Supporting Information. All TGA and DSC figures and tables associated with LS and PS-LS blends are provided as part of the Supporting Information.

\section{References}

Ayoub, A., Venditti, R.A., Jameel, H., Chang, H.-M., 2014. Effect of irradiation on the composotion and thermal properties of softwood kraft lignin and styrene grafted lignin. J Appl Polym Sci. 131 (1), 1-10.

Cui, C. Z., Sadeghifar, H., Sen, S., Argyropoulos, D. S., 2013. Towards thermoplastics lignin polymers Part II thermal \& polymer characteristics of kraft lignin \& derivatives. Bioresources 8 (1), 864-886. 
Capanema, E. A., Balakshin, M. Y., Kadla, J. F., 2004. A comprehensive approach for quantitative lignin characterization by NMR spectroscopy. J Agr Food Chem. 52 (7), 1850-1860.

Crestini, C., Melone, F., Sette, M., Saladino, R., 2011. Milled Wood Lignin: A Linear Oligomer. Biomacromolecules 12 (11), 3928-3935.

Gosselink, R., De Jong, E., Guran, B., Abächerli, A., 2004. Co-ordination network for ligninstandardisation, production and applications adapted to market requirements (Eurolignin) Ind Crop Prod., 20 (2), 121-129.

Glasser, W. G., Barnett, C. A., Rials, T. G., Saraf, V. P., 1984. Engineering plastics from Lignin II. Characterization of hydroxylalkyl lignin derivatives. J Appl Polym Sci, 29 (5), 1815-1830.

Hulin, L., Husson, E., Bonnet, J.-P., Stevanovic, T., Sarazi, C., 2015. Enzymatic transesterification of kraft lignin with long Acyl chains in ionic liquids. Molecules, 20 (9), $16334-16353$

Hult, E.-L. K., Asikkala, J., Ropponen, J., Wrigstedt, P., Sipilä, J., Poppius-Levlin, K., 2013. Esterified lignin coating as water vapor and oxygen barrier for fiber-based packaging. Holzforschung 67 (8), 899-905.

Kelley, S. S., Glasser, W. G., Ward, T. C., 1988. Engineering plastics from lignin XIV. Characterization of chain extended hydroxypropyl lignins. J Wood Chem Technol, 8 (3)341-359. 
Laurichesse, S., Averous, L., 2014. Chemical modification of lignins: Towards biobased polymers. Progress in Polymer Science, 39, 1266-1290.

Lewis, H. F., Brauns, F. E., Buchanan, M. A., Brookbank, E. B. 1943. Lignin esters of mono-and dibasic aliphatic acids. Industrial \& Engineering Chemistry, 35 (10), 1113-1117.

Pawar, S. N., Edgar, K. J., 2011. Chemical modification of alginates in organic solvent systems. Biomacromolecules, 12 (11), 4095-4103.

Mariotti, N., Wang, X-M., Rodrigue, D., Stevanovic, T. 2014. Combination of esterified kraft lignin and MAPE as coupling agent for bark/HDPE composites. Journal of Materials Science Research, 3 (2), 8-22.

Mariotti, N., Hu, L., Schorr, D., Stevanovic, T., Rodrigue, D., Wang, X.-M., Diouf, P. N., Grenier, D., 2012. New Bio-composites containing industrial lignins. Proceedings of the $55^{\text {th }}$ International Convention of the Society of Wood Science and Technology, August 37-31, Beijing, China

Pietarinen, S., Myllymäki, T., K. Eskelinen, A., 2014. Method for esterifying lignin with at least one fatty acid. WO 2014029919 A1.

Saraf, V. P., Glasser, W. G., 1984. Engineering plastics from lignin III Structure property relationships in solution cast polyurethane films. J Appl Polym Sci., 29 (5), 1831-1841. 
Saraf, V. P., Glasser, W. G., Wilkes, G. L., McGrath, J. E., 1985. Engineering plastics from lignin VI Structure-property relationships of PEG containing polyurethane networks. J Appl Polym Sci, 30 (5), 2207-2224.

Sagar, A.D., Merril, E. W., 1995. Properties of fatty-acid esters of starch. J Appl Polym Sci, 58, (9), 1647-1656.

Vishtal, A., Kraslawski, A., 2011. Challenges in industrial applications of technical lignins, Bioresources, 6 (3), 3547-3568.

Wu, L. C. F., Glasser, W. G., 1984. Engineering plastics from lignin I Synthesis of hydroxylpropyl lignin. J Appl Polym Sci. 29 (4), 1111-1123. 
Table 1. Amounts of reagents in reaction, corresponding DS and product solubilities

\begin{tabular}{|c|c|c|c|c|c|c|c|c|c|}
\hline Sample & $\begin{array}{l}\text { St-Cl } \\
\text { eq. } \\
\text { added } \\
\text { per } \\
\text { OH }\end{array}$ & $\begin{array}{c}\text { Pyr } \\
\text { eq. } \\
\text { added } \\
\text { per } \\
\text { OH }\end{array}$ & $\begin{array}{c}\text { DS per } \\
100 \\
\text { aromatic } \\
\text { rings }\end{array}$ & $\% \mathrm{DS}$ & $\mathbf{H}$ & $\mathbf{C}$ & $\mathbf{E}$ & D & W \\
\hline LS-7\% & 0.30 & 0 & 6.09 & 7 & I & I & $\mathrm{S}$ & $\mathrm{S}$ & I \\
\hline LS-13\% & 0.59 & 0 & 12.29 & 13 & I & I & S & S & I \\
\hline LS-46\% & 1.18 & 0 & 43.43 & 46 & S & S & I & I & I \\
\hline LS-90\% & 2.36 & 0 & 84.40 & 90 & S & S & I & I & I \\
\hline LS-95\% & 2.36 & 0.12 & 89.28 & 95 & S & S & I & I & I \\
\hline LS-97\% & 2.36 & 0.50 & 90.72 & 97 & S & S & I & I & I \\
\hline
\end{tabular}

Soluble - S; Insoluble - I

$\mathrm{H}$ - Hexane; C - Chloroform; E - Ethanol; D - DMSO; W - Water 
Table 2: Total moisture content and mass loss measured using TGA and gravimetry. Residual mass by calculation was determined using the mass fractions of fatty acid chains relative to the mass of lignin. Residual mass of the fatty acid was assumed to be $0 \%$, whereas that of lignin was assumed to be $42.67 \%$ (as obtained via TGA analysis of pure lignin) for the calculation and a linear mixing rule assumed.

\begin{tabular}{|llll|} 
Sample & $\begin{array}{l}\text { Moisture } \\
\text { Content } \\
\text { By TGA (\%) }\end{array}$ & $\begin{array}{l}\text { Residual } \\
\text { Mass (\%) }\end{array}$ & $\begin{array}{l}\text { Residual TGA } \\
(\%)\end{array}$ \\
& 2.32 & 42.67 & By Calculation \\
\hline BCL & 1.94 & 46.18 & - \\
LS-7\% & 0.94 & 39.38 & 38.93 \\
LS-13\% & 0.66 & 28.24 & 26.20 \\
LS-46\% & 0.0 & 21.04 & 19.04 \\
LS-90\% & 0.0 & 16.82 & 18.46 \\
LS-95\% & 0.0 & 17.50 & 18.25 \\
LS-97\% & & & \\
\hline
\end{tabular}


Table 3: $T_{g}$ and $\Delta C_{p}$ values for PS blends with BCL, LS-46\% and LS-97\% measure by DSC in the $2^{\text {nd }}$ heating scan. Melting point onset is indicated for the $1^{\text {st }}$ heating scan.

\begin{tabular}{|llll|} 
Sample & $\mathbf{T}_{\mathbf{g}}\left({ }^{\circ} \mathbf{C}\right)$ & $\Delta \mathbf{C}_{\mathbf{p}}\left(\mathbf{J} / \mathbf{g} /{ }^{\circ} \mathbf{C}\right)$ & $\mathbf{M P}\left({ }^{\circ} \mathbf{C}\right)$ \\
\hline PS & 99 & 0.242 & - \\
BCL & 144 & 0.31 & - \\
LS97\%PS5\% & 98 & 0.237 & - \\
LS97\%PS25\% & 78 & 0.218 & - \\
LS97\%PS100\% & - & - & 46 \\
LS46\%PS5\% & 88 & 0.252 & - \\
LS46\%PS25\% & 91 & 0.193 & - \\
LS46\%PS100\% & - & - & 53 \\
BCL PS5\% & 101 & 0.277 & - \\
BCL PS25\% & 96 & 0.231 & - \\
BCL PS100\% & 135 & 0.212 & - \\
\hline
\end{tabular}


Figure 1

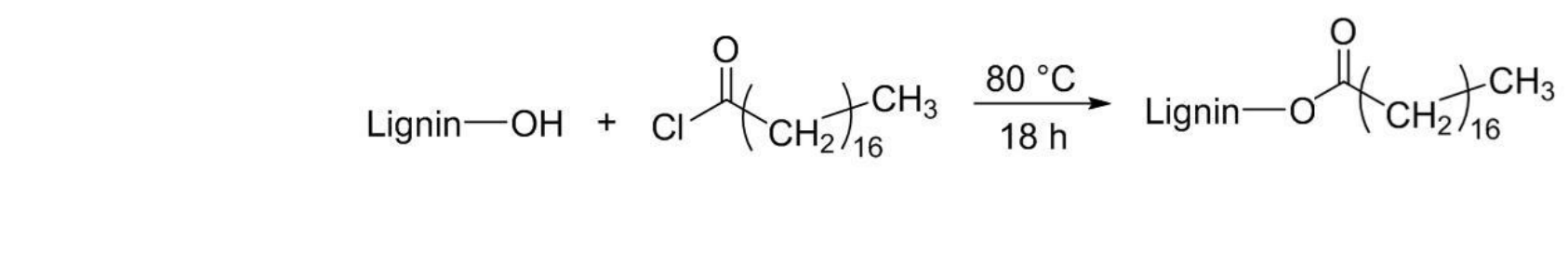

Figure 1. Reaction scheme for LS synthesis

Figure 1. Reaction scheme for LS synthesis

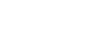

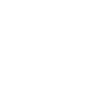$$
\text { ( }
$$

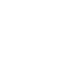

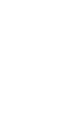

.

(2)
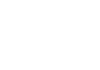
Figure 2

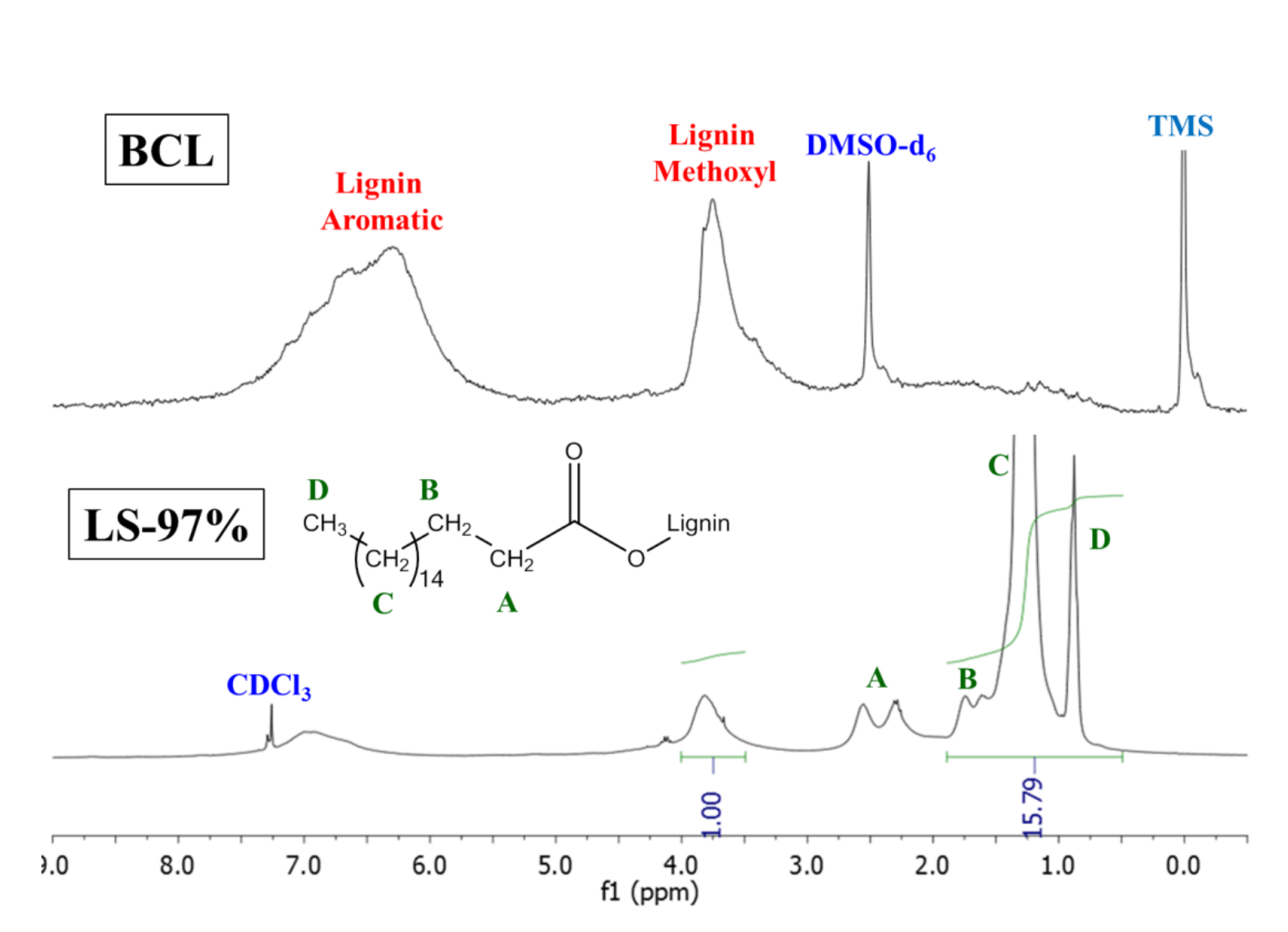

Figure 2. ${ }^{1} \mathrm{H}-\mathrm{NMR}$ spectra of BCL and LS-97\%

\section{Figure 2}

BCI

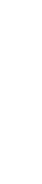

.

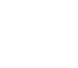

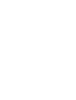

$x^{2}$

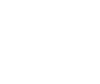

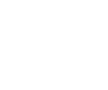


Figure 3

\section{BCL}
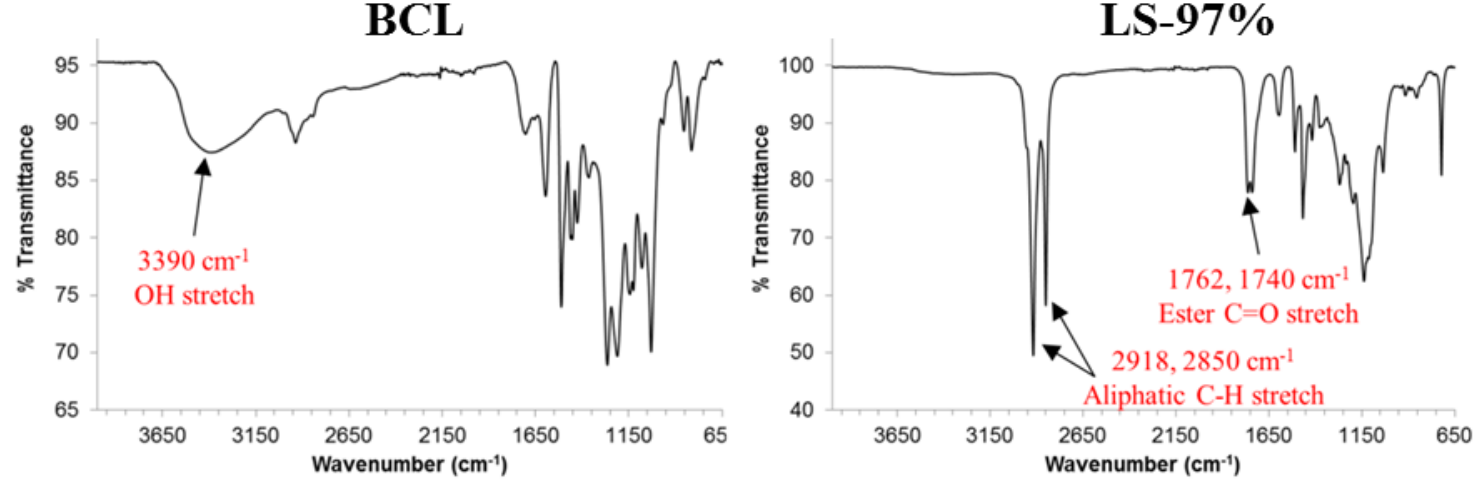

Figure 3. FTIR spectra of BCL and LS-97\% 
Figure 4
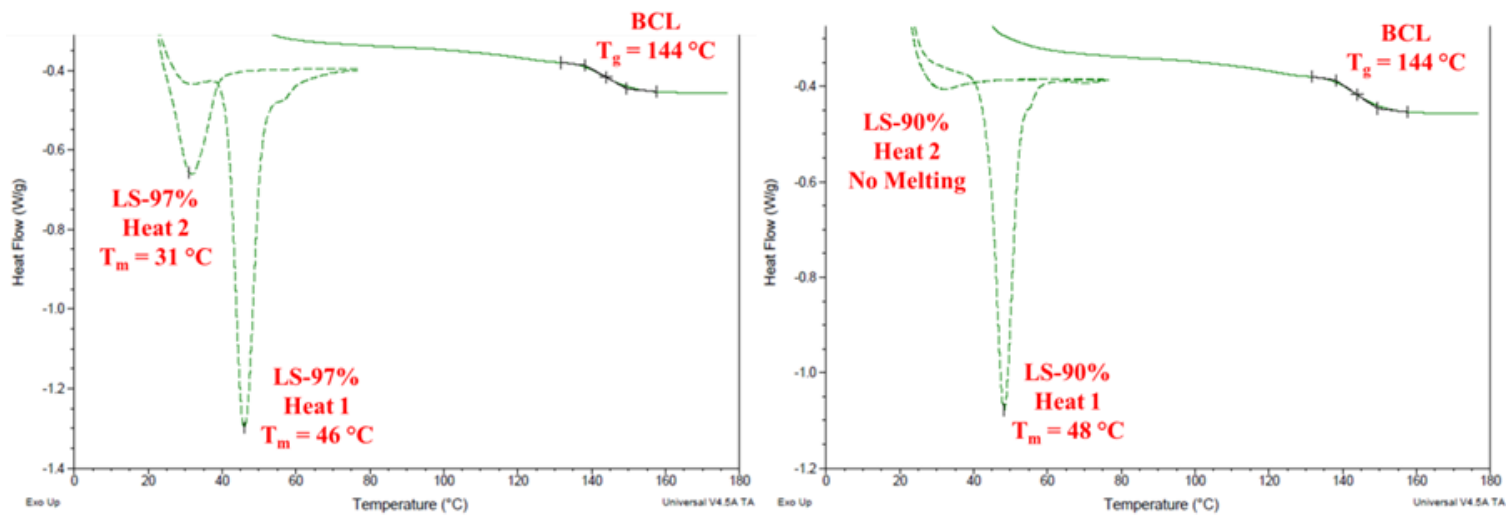

Figure 4. DSC thermograms of BCL compared to LS-90\% and LS-97\% 
Figure 5

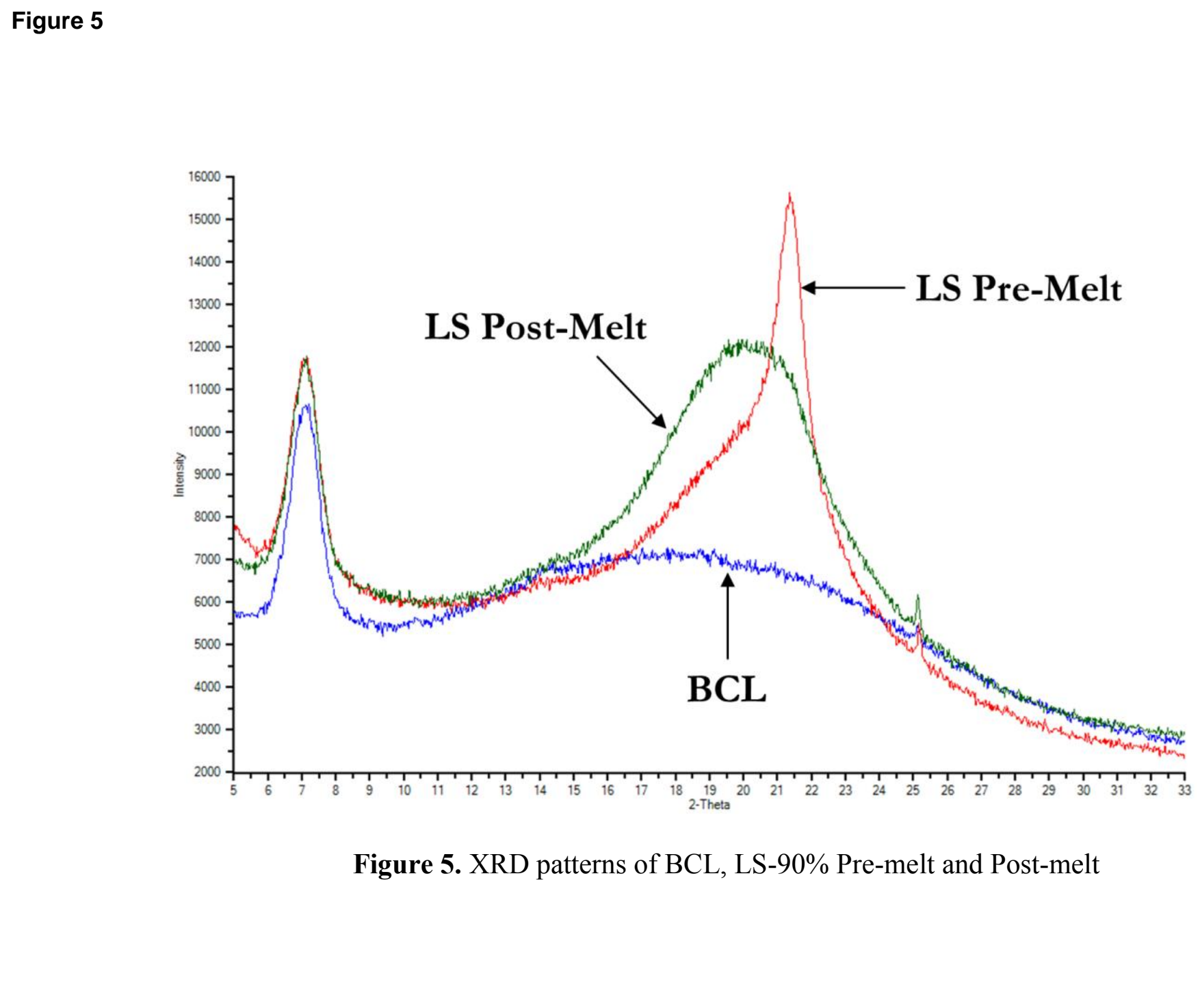

Figure 5. XRD patterns of BCL, LS-90\% Pre-melt and Post-melt

\author{
(n)
}

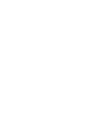
-
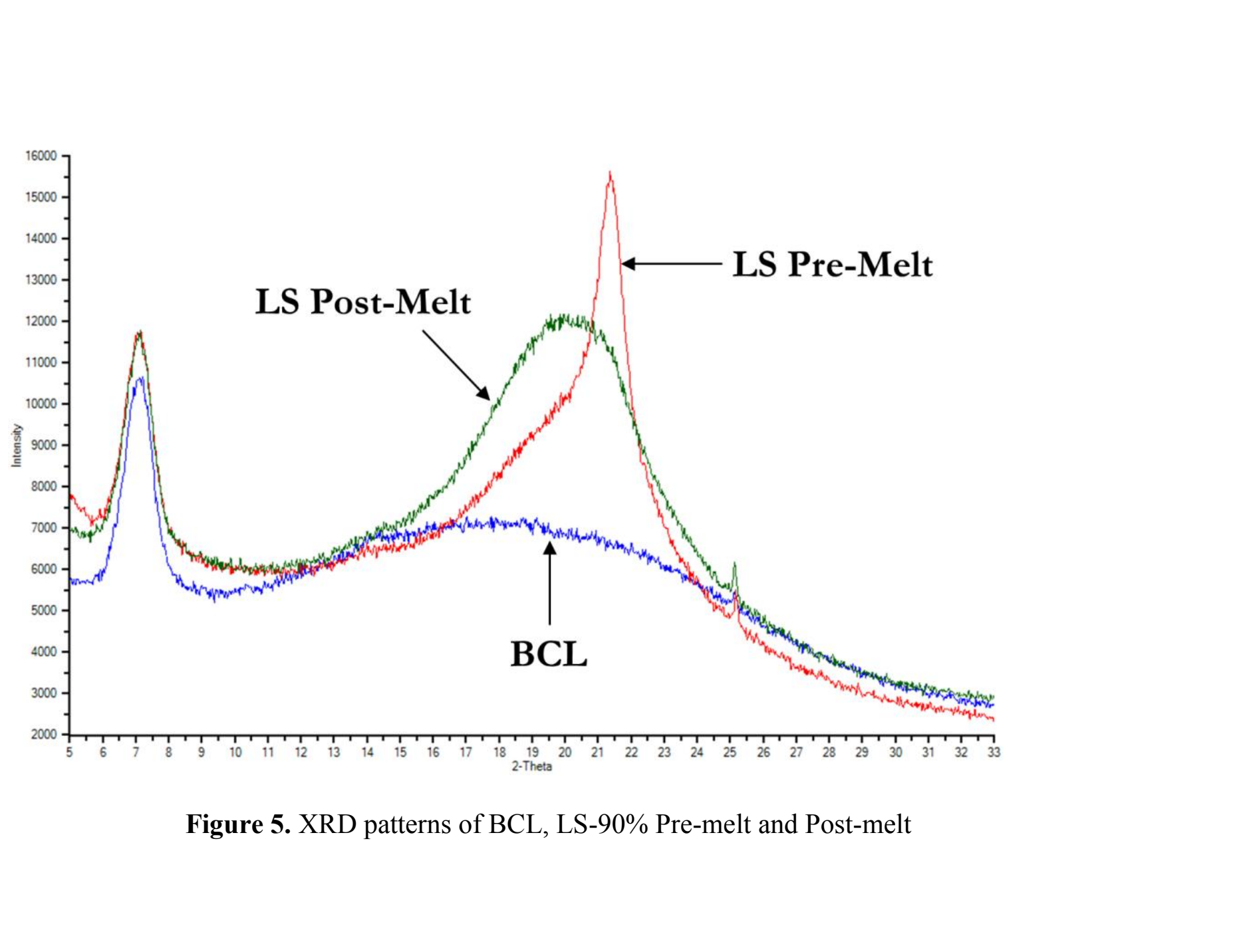

(1)

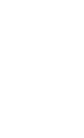



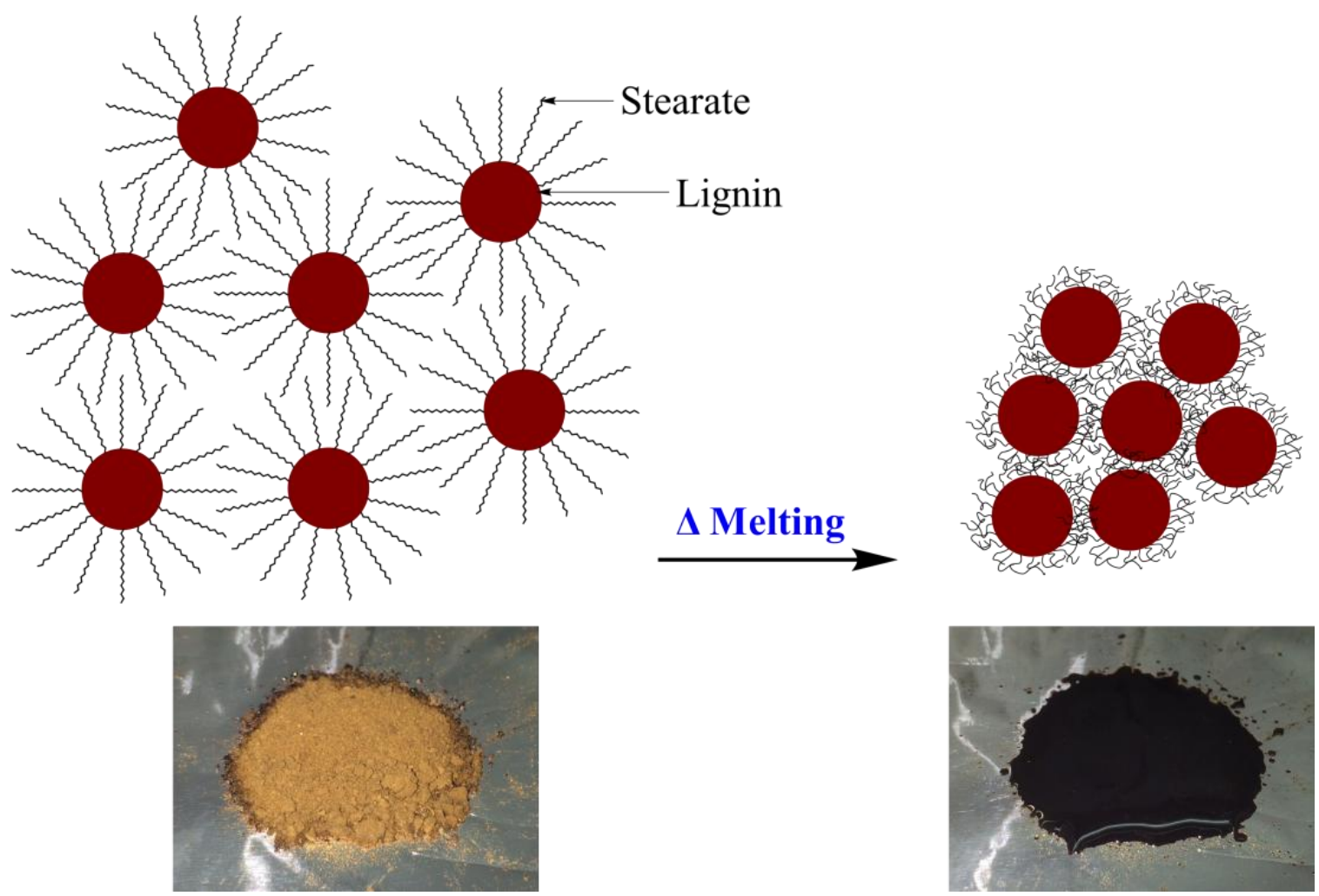

Figure 6. Schematic representation (top) and images (bottom) of LS-90\% before and after melting 

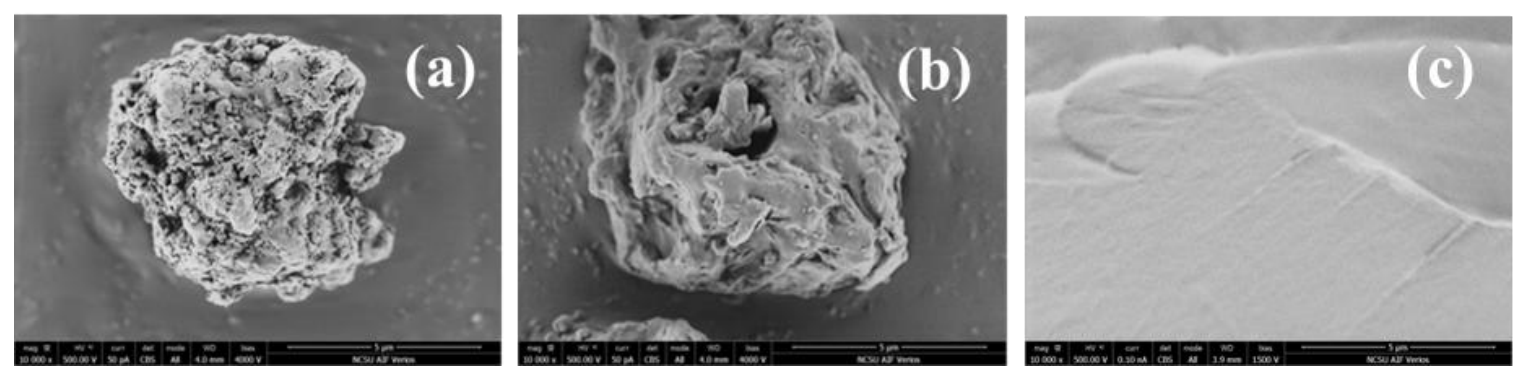

Figure 7. SEM images of (a) BCL, (b) LS-90\% Pre-Melt and (c) LS-90\% Post-Melt

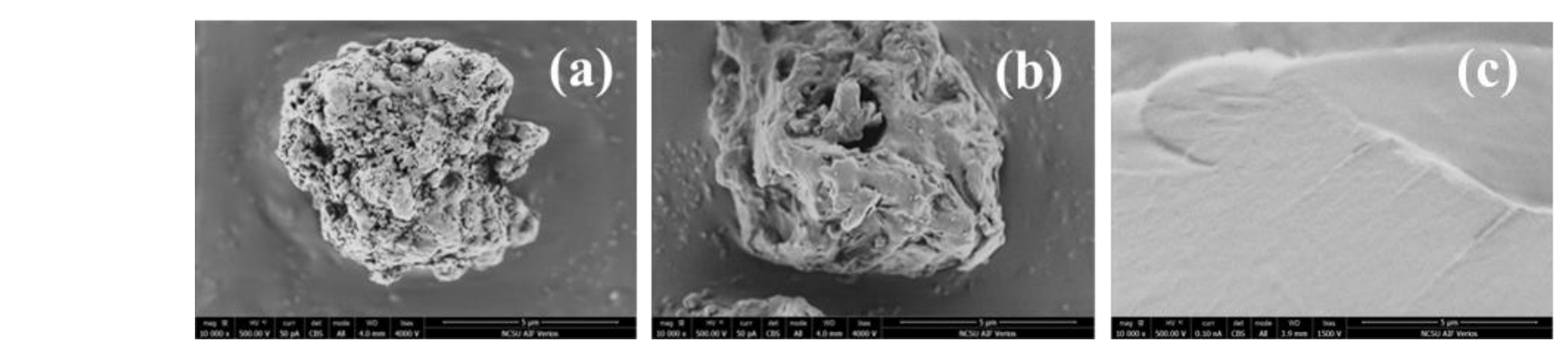

(a) 